\title{
Ralf Buckley, Adventure Tourism Management
}

Elsevier, 2010, 268 pages

\section{Pascal Mao}

\section{OpenEdition \\ Journals}

Édition électronique

URL : http://journals.openedition.org/tourisme/298

DOI : 10.4000/tourisme.298

ISSN : 2492-7503

Éditeur

Éditions touristiques européennes

Édition imprimée

Date de publication : 1 décembre 2010

Pagination : 115-116

ISSN : 2109-5671

Référence électronique

Pascal Mao, «Ralf Buckley, Adventure Tourism Management », Mondes du Tourisme [En ligne], 2 | 2010, mis en ligne le 30 septembre 2015, consulté le 22 septembre 2020. URL : http://

journals.openedition.org/tourisme/298; DOI : https://doi.org/10.4000/tourisme.298

Ce document a été généré automatiquement le 22 septembre 2020.

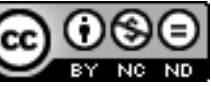

Mondes du tourisme est mis à disposition selon les termes de la licence Creative Commons Attribution

- Pas d'Utilisation Commerciale - Pas de Modification 4.0 International. 


\section{Ralf Buckley, Adventure Tourism Management}

Elsevier, 2010, 268 pages

Pascal Mao

\section{RÉFÉRENCE}

Ralf Buckley, Adventure Tourism Management, coll. "Butterworth-Heinemann”, Elsevier, 2010.

1 Le professeur Ralf Buckey est directeur du centre international de recherches sur l'écotourisme de l'Université Griffith, en Australie. On lui doit de nombreuses contributions scientifiques sur la gestion, l'aménagement et le développement d'activités récréatives et touristiques dans des espaces naturels : près de 200 articles et 12 ouvrages portant essentiellement sur l'écotourisme, l'impact des pratiques sur l'environnement, la gestion des milieux et l'effet du changement climatique sur les destinations touristiques.

2 L'angle d'analyse privilégié ici par l'auteur est une approche marketing des produits et des marchés du tourisme d'aventure. Cet ouvrage fait suite à une publication collective qu'il a dirigée en 2006 sur le même thème (éd. CAB international, Wallingford). Le livre est organisé en 4 parties et 13 chapitres thématiques. Ces derniers sont systématiquement organisés sur le même modèle, présentant successivement: des mots clés, un résumé, une revue de littérature détaillée mobilisant plus de 500 références exclusivement anglophones, des illustrations via des études de cas ou une analyse de produits et enfin, une synthèse conclusive. Ce choix de présentation permet au lecteur d'aborder chaque chapitre ou thématique de manière parfaitement autonome. Cet ouvrage est à destination tant des agents économiques que des chercheurs intéressés par la question.

3 La partie 1 propose une définition du champ thématique concerné par le tourisme d'aventure et une présentation des singularités de fonctionnement du marché (en 
termes d'offre et de demande). La partie 2 s'intéresse au management opérationnel des produits touristiques par les opérateurs afin de gérer les questions de risques, de communication, d'accès aux espaces, d'impact environnemental et d'adaptation au changement climatique. La partie 3 propose une analyse des secteurs d'activités représentatifs, selon l'auteur, du tourisme d'aventure. Sont ainsi successivement analysés l'observation de la vie sauvage, les pratiques nautiques, les sports de glisse et l'héliski. La partie 4 vient conclure le propos en offrant une analyse prospective en lien avec ce secteur d'activités.

4 Cette approche du tourisme d'aventure peut questionner dans sa délimitation même de l'objet étudié, d'un point de vue tant géographique que des pratiques, lieux ou produits concernés. La volonté d'avoir une vision mondiale du phénomène impose de traiter indifféremment des zones polaires, océaniques, désertiques, côtières, etc., ainsi qu'une offre d'activités extrêmement large, qui peut être perçue a priori comme hétéroclite. Pour ne prendre que quelques exemples, quels liens existe-il entre du kayak de mer en Terre de Baffin, la pratique du snowboard dans la station de Niseko au Japon, la pratique de l'héliski dans les Rocheuses canadiennes, une randonnée d'observation de la faune sauvage dans un parc naturel au Costa Rica ou encore un séjour sur un paquebot de croisière en Antarctique afin d'observer des baleines? Cela renvoie à la définition même de la notion d'aventure. Le point commun entre ces différents exemples, comme le définit l'auteur en introduction (p.6), est que le tourisme d'aventure concerne uniquement les activités commerciales encadrées dont le principal intérêt repose sur des activités d'outdoor, en lien avec la découverte d'espaces naturels. De fait, cela renvoie aux pratiques de l'outdoor et à l'interprétation pour le moins extensive retenue par les chercheurs anglophones qui y intègrent (p.5) les raids motorisés, le yachting, la voltige aérienne comme le trekking, l'alpinisme ou l'équitation.

5 Pour conclure, cet ouvrage ouvre une perspective large sur une thématique et des problématiques peu représentées dans la littérature francophone. La bibliographie proposée par l'auteur, extrêmement large, invite le lecteur curieux à étendre ses investigations. 